\title{
Thrombophilia and cardioembolic stroke in a young patient. A case report
}

\author{
Dana Simona CHITA ${ }^{1}$, Ana Maria PAH ${ }^{2,3}$, Nicoleta Florina BULEU ${ }^{2}$, \\ Simona Ruxanda DRAGAN ${ }^{2,3}$ \\ 1"Victor Babes" University of Medicine and Pharmacy, Timisoara, Romania \\ 2Department of Cardiology, "Victor Babes" University of Medicine and Pharmacy, \\ Timisoara, Romania \\ ${ }^{3}$ Institute of Cardiovascular Diseases, Timisoara, Romania
}

\begin{abstract}
Thrombophilia is well known to be an important risk factor for venous thrombosis, but there is not enough clinical data to explain the association with arterial thrombosis. While there are not sufficient and consistent clinical studies to demonstrate an association between thrombophilia and cardioembolic stroke, several mutations in the methylenetetrahydrofolate reductase gene (MTHFR) have been studied as risk factors for conditions like ischemic stroke, coronary artery disease, diabetes mellitus and more. However, data regarding mutations in the MTHFR gene are insufficient for cardioembolic stroke. We report a case of cardioembolic stroke associated with thrombophilia (with MTHFR A1298C mutation) in a young patient. The co-morbid states (non-valvular AF, carotid atheromatosis, cardioembolic stroke) associated with thrombophilia, but also stroke severity and clinical evolution of this patient are a case particularity.
\end{abstract}

Keywords: thrombophilia, cardioembolic stroke, MTHFR, non-valvular AF, carotid atheromatosis

\section{INTRODUCTION}

Thrombophilia is a well-recognized risk factor for venous thrombosis, but an association between it and arterial thrombosis has not been clearly established. There are also various other acquired conditions that are associated with this hypercoagulable state. Neither case-control studies, nor meta-analyzes published so far were consistent in analyzing the complex relationship between thrombophilia and cardioembolic stroke (1). A rare autosomal recessive disease, methylenetetrahydrofolate reductase (MTHFR) deficiency, affects intracellular folate metabolism with impairment of various organ systems and with clinical mani- festations including heart disease, fetal neural tube defects, schizophrenia, autism, poor pregnancy outcomes and colorectal cancer (2). C677T polymorphism in the MTHFR gene has shown inconsistent results in clinical studies when associated with stroke, most likely due to small sample sizes and different types of strokes included (3). In a retrospective case-control study that evaluated the association between these polymorphisms and ischemic stroke the authors observed that an increased risk of ischemic stroke was only associated with the homozygous TT genotype, even after adjustment for conventional risk factors (4). We report a case of cardioembolic stroke associated with thrombophilia. 


\section{CASE PRESENTATION}

A 48 years old male patient with a history of acute ischemic stroke in 2018, hospitalized at the Neurology Department of "Pius Branzeu" Emergency Clinical Hospital in Timisoara, presented in May 2020 in our clinic for clinical reevaluation. As cardiovascular risk factors involved in ischemic stroke, he had chronic hypertension, treated prior to hospitalization for 6 years, a smoking habit for 20 years and he was overweight.

\section{Past clinical history}

The patient presented right hemiparesis, dysarthria with elements of Broca aphasia (difficulties in naming objects and using words correctly) and gait disturbance on admission. The clinical onset was 2 days before hospitalization for the language deficit and 1 day for the onset of motor deficit. Due to late hospital presentation, thrombolytic therapy was not recommended.

The general clinical examination revealed high blood pressure ( $B P=170 / 90 \mathrm{mmHg}$, heart rate $H R=60$ $\mathrm{bpm})$ and overweight, with body mass index (BMI = $\left.28.3 \mathrm{~kg} / \mathrm{m}^{2}\right)$ and normal clinical status for cardiac, pulmonary $\left(\mathrm{O}_{2}\right.$ saturation $\left.=98 \%\right)$, abdominal and renal functions. The neurological examination on admission revealed right ataxic hemiparesis with right-sided deep tendon hyperreflexia and positive right-sided Babinski sign, right limb ataxia with dysmetria, ataxic gait, no sign of objective sensibility disturbances, normal mental status (alert, attentive and oriented), no neck muscle rigidity and meningeal signs. The clinical assessment of cranial nerves revealed right central facial palsy with no other dysfunctions of the cranial nerves. When assessing language features, dysarthria with Broca aphasia elements were observed.

The patient underwent blood testing and several clinical investigations. The laboratory findings performed during hospitalization revealed a normal hemoleucogram, slight hyperglycemia on admission $(117 \mathrm{mg} / \mathrm{dl})$, normal lipid profile with total cholesterol ( $\mathrm{TC}=129 \mathrm{mg} / \mathrm{dl}$ ), high density lipoprotein cholesterol $(\mathrm{HDLC}=28 \mathrm{mg} / \mathrm{dl})$, low density lipoprotein cholesterol $(\mathrm{LDLC}=81 \mathrm{mg} / \mathrm{dl})$ and triglicerides $(\mathrm{TGL}=78 \mathrm{mg} / \mathrm{dl})$, normal coagulation tests (APTT $=31.5 \mathrm{~s} ; \mathrm{TQ}=11.5 \mathrm{~s}$ ) with INR (international normalized ratio) values ranging from 1.05 on admission to 1.92 at hospital leave, hyperfibrogenemia $(373 \mathrm{mg} / \mathrm{dl})$, slightly elevated muscle creatine phosphokinase $(\mathrm{CK}-\mathrm{MB}=11 \mathrm{U} / \mathrm{I})$ and serum lactate-dehydrogenase $(\mathrm{LDH}=138 \mathrm{mg} / \mathrm{dl})$ and normal C-reactive protein (CRP $=3.27 \mathrm{mg} / \mathrm{l})$.

The electrocardiogram revealed sinus rhythm, but on the $24 \mathrm{~h}$ Holter ECG monitoring an episode of paroxysmal AF was found with a heart rate of $95 \mathrm{~b} / \mathrm{min}$. The in-hospital echocardiographic parameters were normal with no left atrial or ventricular hypertrophy, while valvulopathies were also excluded during this examination. The ejection fraction of the left ventricle was normal (LVEF $=65 \%$ ).

Bilateral diffuse carotid atheromatosis with increased intima media thickness (IMT $=1.2 \mathrm{~mm}$ ) was found on Doppler carotid echography. Also an abdominal echography was performed with no modifications. The chest X-ray showed diffuse scleroemphysema and bilateral millimetrical fibronodular opacities in the middle lobes.

On admission a cerebral computed tomography scan was performed revealing a frontal left hypodense lesion with a diameter of $26 \mathrm{~mm}$ and no signs of intracranial bleeding. Also native and contrast enhanced magnetic resonance imaging (MRI) cerebral studies were performed that highlighted the presence of multiple cortical and subcortical acute ischemic lesions (the biggest lesion with a diameter of $20 \mathrm{~mm}$ ) with localization in the left parietal lobe, left operculum and left insula (figures 1 and 2). The ventricles were normally sized with normal alignment. There were also no pathologic cerebral contrast captions.

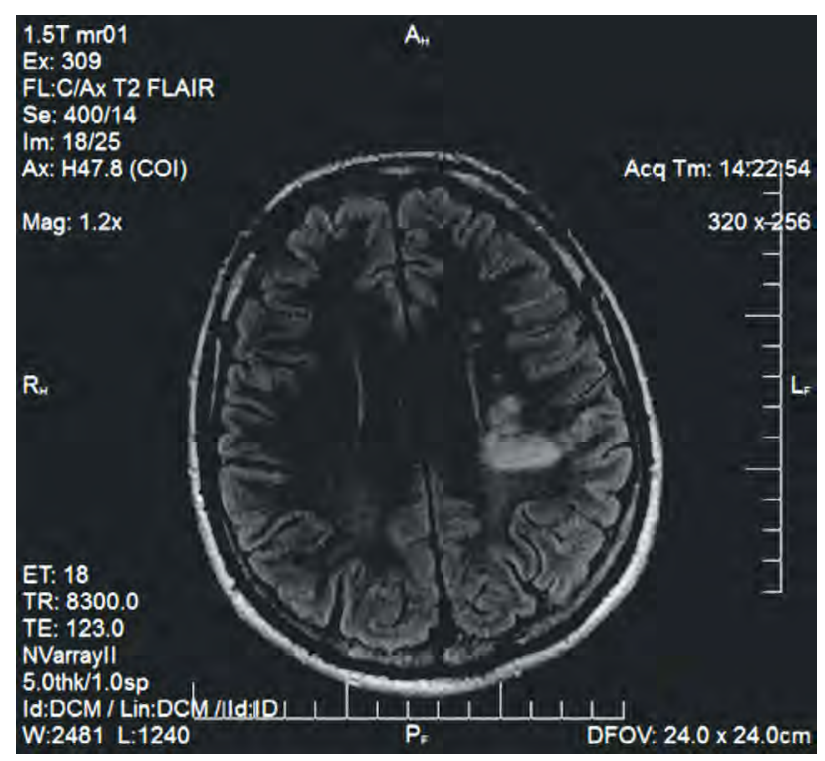

FIGURE 1. Axial T2 FLAIR weighted MRI scan image showing multiple cortico-subcortical hyperintensities in the left parietal lobe, left parietal operculum and left insula with the biggest diameter of $20 \mathrm{~mm}$

The $\mathrm{CHA}_{2} \mathrm{DS}_{2}$-VASC score calculated during hospitalization was of 2 points, being relatively low and the HASBLED score was 1 point. Stroke severity scales were applied at admission, the NIHSS scale was 8 and the modified Rankin scale was 3.

Further investigations were made to determine other possible etiologies of ischemic stroke for this relatively young patient, and a genetic profile for thrombophilia was assessed. The results showed a heterozygous factor II G20210A mutation, PAI-1 4G/4G 
homozygous mutation, methylenetetrahydrofolate reductase MTHFR A1298C heterozygous mutation and EPCR (endothelial protein $C$ receptor) also present with alleles A1/A2. The other components of this genetic profile showed no signs of mutations, respectively the factor $\mathrm{V}$ (Leiden) G1691A mutation, factor $\mathrm{V}$ (H1299R) mutation, MTHFR C677T mutation and factor XIII V34L mutation were absent. Slightly elevated plasma homocysteine levels (tHcy) were found, respectively $11.3 \mu \mathrm{mol} / \mathrm{l}$.

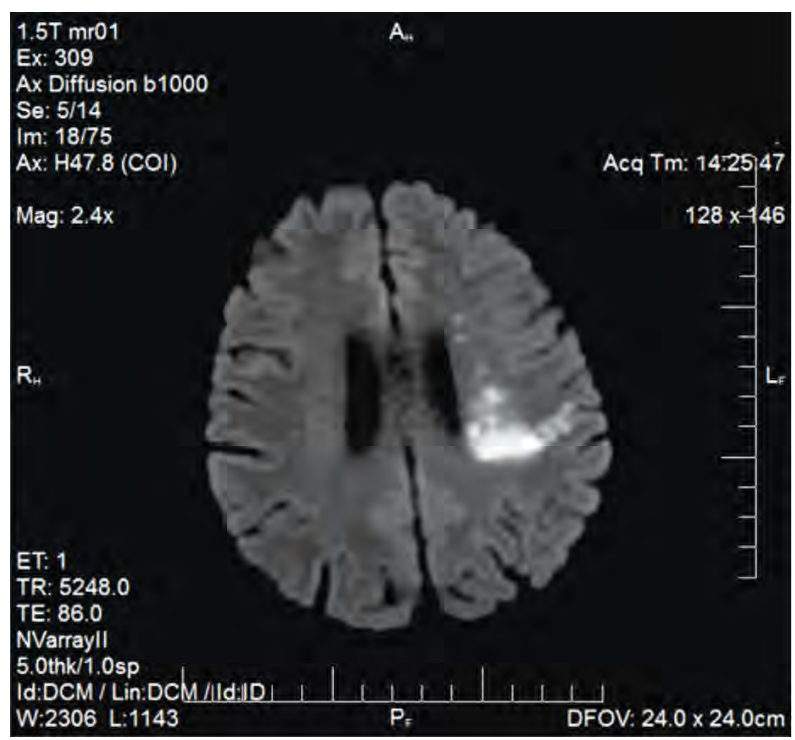

FIGURE 2. The same image in axial diffusion MRI scan revealing multiple cortico-subcortical hyperintensities in the left parietal lobe, operculum and insula

The in-hospital clinical diagnosis was multiple infarct ischemic stroke, right ataxic hemiparesis, dysarthria, thrombophilia with homozygous PAI-1 4G/4G, heterozygous MTHFR A1298C and factor II G20210A heterozygous mutation, paroxysmal NVAF and arterial hypertension. The patient received antiplatelet therapy (Aspirin), vasodilators, lipid lowering therapy and neurotropic medication during the hospital stay.

The clinical evolution was favorable with a slight improvement on neurological examination, the right-sided motor deficit remained predominantly in the right upper limb and dysarthria was also slightly improved on hospital leave.

After a few months of combined drug therapy, physical therapy and speech therapy, the clinical status of the patient improved gradually and until present time he had no other hospital admissions.

\section{Present clinical status}

On admission in our clinic we performed a general and neurological examination of the patient. BMI, blood pressure and heart rate were normal. The neurological exam showed a right-sided pyramidal syndrome with brisk deep tendon reflexes on the right and right sided positive Babinski sign. Language when assessed was normal with no signs of dysarthria. ECG results showed no signs of ischemia or modifications of heart waves or intervals. The current medication of the patient includes oral anticoagulation therapy (Acenocoumarol) under INR control, hypotensive agents such as Indapamid ( $1.5 \mathrm{mg} /$ day) and Telmisar$\tan (80 \mathrm{mg} /$ day), heart rate regulator Nebivolol $(5 \mathrm{mg} /$ day) and the lipid-lowering agent Atorvastatin $(20 \mathrm{mg} /$ day).

\section{DISCUSSIONS}

The presented case associates complex co-morbidities such as multiple infarct ischemic stroke, paroxysmal NVAF, carotid atheromatosis and thrombophilia in a relatively young patient. The exact etiology of ischemic stroke in this case is very hard to determine, due to the multiple causative pathologies. On one hand we can suspect that the prior undiscovered and untreated thrombophilia with homozygous mutation in the PAI-1 4G/4G gene and the heterozygous mutations in the MTHFR A1298C and factor II G20210A could be the main causes of ischemic stroke in this case, leading to elevated plasma levels of tHcy. It has been studied that increased homocysteine plasma levels are associated with cardiac and cerebrovascular diseases but also with other vascular pathologies such as peripheral artery disease and atherosclerosis, due to the accelerated damage in the endothelial cells leading to thrombosis. [5]. Mutations in the MTHFR gene are a common cause for increased tHcy levels, especially the homozygous C677T mutation [6]. Also a meta-analysis performed by Kang S. et al. [7] revealed that the presence of the $\mathrm{A} 1298 \mathrm{C}$ mutation in the MTHFR gene is linked to an increased ischemic stroke risk, especially the $\mathrm{C}$ allele. The clinical implications of these inherited genetic variants of the MTHFR gene have been studied in numerous diseases, but regarding cardioembolic stroke and atrial fibrillation there is a lack of clinical data. However, some studies managed to find an association between the C677T mutation and lacunar stroke in hypertensive patients [8]. Also Taveira A. et al. presented a case of a relatively young female (46 years) with thrombophilia and striato-capsular ischemic stroke and they concluded that the most plausible origin of stroke was embolic as consequence of inherited thrombophilia [9], while other investigations proved negative. In our case, the patient is somewhat the same age (48 years), but has multiple risk factors associated (hypertension, smoking, overweight) and also multiple co-morbidities that could determine stroke (paroxysmal AF and carotid atheromatosis). A review by de Lau LM et al. failed to document the causality between ischemic stroke and coag- 
ulation disorders in the studies available in the analyzed timespan [10]. Another controversial study by Giusti B. et al., regarding causality between non-valvular AF (NVAF) and MTHFR gene polymorphisms, also failed to demonstrate an association [11].

\section{CONCLUSIONS}

Despite of inconsistent published data regarding associations between genetic polymorphisms of the MTHFR gene, risk factors and stroke, we can conclude that in this case the complex interconnections between all these factors caused multiple stroke at a relatively young age. Another particularity of the case was the relatively low CHA2DS2-VASC score on admis- sion, despite of the presence of a high thrombotic state, suggesting that in such cases individual risk assessment is needed beyond the usual scoring systems. Also the moderate clinical severity on admission with very good in-hospital and at-home recovery is another case particularity. However, the prognosis for this patient is severe because of the high stroke recurrence risk and the possible evolution toward vascular dementia, even under chronic secondary prevention treatment (oral anticoagulation therapy). All modifiable cardiovascular risk factors (smoking, dyslipidemia, obesity) need to be corrected by persistent and responsible life-style changes.

Conflict of interest: none declared Financial support: none declared

\section{REFERENCES}

1. Chiasakul T, Jesus ED, Tong J, Chen $Y$, Crowther M, Garcia D et al. Inherited Thrombophilia and the Risk of Arterial Ischemic Stroke: A Systematic Review and Meta\&\#x2010;Analysis. Journal of the American Heart Association 2019;8(19):e012877.

2. Long S, Goldblatt J. MTHFR genetic testing: Controversy and clinical implications. Aust Fam Physician 2016;45(4):237-40.

3. Arsene D, Gaina G, Balescu C, Ardeleanu C. C677T and A1298C methylenetetrahydropholate reductase (MTHFR) polymorphisms as factors involved in ischemic stroke. Romanian Journal of Morphology and Embryology 2011;52(4):1203-7.

4. Hou J, Zeng X, Xie Y, Wu H, Zhao P. Genetic polymorphisms of methylenetetrahydrofolate reductase
C677T and risk of ischemic stroke in a southern Chinese Hakka population. Medicine 2018;97(51):e13645-e45.

5. Fanapour PC, Yug B, Kochar MS. Hyperhomocysteinemia: An additional cardiovascular risk factor. WMJ (official publication of the State Medical Society of Wisconsin) 1999;98(8):51-4.

6. Reilly R, McNulty H, Pentieva K, Strain JJ, Ward M. MTHFR 677TT genotype and disease risk: Is there a modulating role for B-vitamins? The Proceedings of the Nutrition Society 2014;73(1):47-56.

7. Kang S, Wu Y, Liu L, Zhao X, Zhang D. Association of the $A 1298 \mathrm{C}$ polymorphism in MTHFR gene with ischemic stroke. Journal of Clinical Neuroscience 2014;21(2):198202.

8. Rutten-Jacobs LC, Traylor M, Adib-Samii P, Thijs V, Sudlow C, Rothwell PM et al. Association of MTHFR C677T Genotype
With Ischemic Stroke Is Confined to Cerebral Small Vessel Disease Subtype. Stroke 2016;47(3):646-51.

9. Taveira I, Vicente C, Sobral S, Nzwalo H, Costa JSE. Multiple Inherited Thrombophilias in a Young Patient with Striatocapsular Stroke. Journal of Neurosciences In Rural Practice 2017;8(Suppl 1): S154-s55.

10. de Lau LM, Leebeek FW, de Maat MP, Koudstaal PJ, Dippel DW. A review of hereditary and acquired coagulation disorders in the aetiology of ischaemic stroke. International Journal of Stroke 2010;5(5):385-94.

11. Giusti B, Gori AM, Marcucci R, Sestini I, Saracini C, Sticchi E et al. Role of C677T and A1298C MTHFR, A2756G MTR and $-786 \mathrm{C} / \mathrm{T}$ eNOS gene polymorphisms in atrial fibrillation susceptibility. PloS One 2007;2(6):e495-e95. 